\title{
Local Linear Neighbor Reconstruction for Multi-view Data
}

\author{
Linlin Zong ${ }^{\mathrm{a}, \mathrm{b}}$, Xianchao Zhang ${ }^{\mathrm{b}, * *}$, Hong Yu ${ }^{\mathrm{b}}$, Qianli Zhao ${ }^{\mathrm{a}, \mathrm{b}}$, Feng Ding ${ }^{\mathrm{b}}$ \\ ${ }^{a}$ Faculty of Electronic Information and Electrical Engineering, Dalian University of Technology, Dalian, China \\ ${ }^{b}$ School of Software, Dalian University of Technology, Dalian, China
}

\section{ABSTRACT}

Graph based multi-view data analysis has become a hot topic in the past decade, and multi-view similarity matrix is fundamental for such tasks. Existing multi-view similarity matrix construction methods can not learn local geometrical information in the original data space from multiple views simultaneously. Considering the fact that an appropriate similarity matrix is block-wise with intra-class similarity, it is more reasonable to learn a similarity matrix by using local geometrical information in multiple original data space. In this paper, we propose to construct a unified similarity matrix by using local linear neighbors in multiple views. In each view, the similarity matrix can be reconstructed with the weights of the neighbors of each data point in the original space. In multiple views, we seek for a unified similarity matrix which consists of the similarity matrix in each view. The unified similarity matrix can be used for spectral clustering, label propagation and other graph based learning algorithms. Experimental results show that spectral clustering and label propagation algorithms using the unified similarity matrix outperform those using other multi-view similarity matrices, they also outperform typical multi-view spectral clustering algorithms and typical multi-view label propagation algorithms.

Keyword: Multi-view similarity, Similarity construction, Local linear neighbor

(c) 2016 Elsevier Ltd. All rights reserved.

\section{Introduction}

Real-world datasets are often collected from multiple views which are compatible and complementary. For example, journalists may report the same news in different ways. A webpage can be described by its content and the hyperlinks linking to it. An object can be photographed by several monitors. Multi-view data analysis has become a hot topic in the past decade and many algorithms have been proposed $[4,5,27,12,26,25,13,38,29]$.

Multi-view similarity matrix is fundamental for graph based multi-view data analysis such as spectral clustering [32] aiming to partition a dataset into groups according to the pairwise similarities between data points, and label propagation [47] predicting the labels of the unlabeled points according to the labeled

\footnotetext{
${ }^{* *}$ Corresponding author. Tel.: +86 411 84709360; fax: +86 41184709360 e-mail: zonglinlin324@gmail.com (Linlin Zong), xczhang@dlut.edu.cn (Xianchao Zhang), hongyu@dlut.edu.cn (Hong Yu), sconan32@live.com (Qianli Zhao), dingfeng@dlut.edu.cn (Feng Ding)
}

points and similarity matrix. Recently, several algorithms have been proposed to construct similarity matrix by integrating information from multiple views. Kumar and Daum [19] constructed the combined similarity matrix in one view by using the eigenvectors in another view. Bisson and Grimal [6] simultaneously handled all the information contained in multi-view datasets by using several instances of an existing co-similarity algorithm which transforms the global data matrix into a similarity matrix. Xia et al. [35] conducted Markov chain based spectral clustering and learned the consensus transition probability matrix via low-rank and sparse decomposition. Multiple kernel learning algorithms $[30,15]$ learned a combined kernel as a function from the input kernels. Tzortzis and Likas [30] learned the coefficient of the linear combination by minimizing the objective function values of multiple views. Guo et al. [15] learned a linear combination of multiple kernels and determined the coefficient by minimizing the kernel alignment between the learned kernel and the kernel of each view. De Sa et al. [14] generated a multipartite graph based on the Minimizing-Disagreement algorithm [25, 13]. Li et al. [22] approximated the similarity graphs using bipartite graphs. How- 
ever, these multi-view similarity matrix construction method$\mathrm{s}$ can not learn local geometrical information in the original data space from multiple views simultaneously. Considering the fact that an appropriate similarity matrix is block-wise with intra-class similarity, it is more reasonable to learn the similarity matrix by using local geometrical information. As shown in [24], the local geometry can be characterized by the local linear neighbors. Recently, many methods have been proposed to construct similarity matrix by using local linear neighbors $[24,33,44,8,43]$. However, those methods are designed for single-view data. In addition, learning from the multiple original data spaces will reduce the information loss in the process of constructing multi-view similarity matrix.

In this paper, we propose to construct a unified similarity matrix by using local linear neighbors in multiple original spaces. In each view, the similarity matrix can be reconstructed with the weight of neighbors of each data point in the original data space $[24,44]$. Intuitively, for the same set of data points with multiple views, the similarity matrix in each view should consist with a ground truth similarity matrix. Thus we seek for an approximated ground truth similarity matrix that is reconstructed with neighbors in multiple original data spaces and consistent with the similarity matrix in each view. The unified similarity matrix and the single-view similarity matrix are learned iteratively. The unified similarity matrix can be used for spectral clustering, label propagation and other graph based algorithms. Experimental results show that spectral clustering and label propagation algorithms using the learned similarity matrix outperform those using other multi-view similarity matrices, they also outperform typical multi-view spectral clustering algorithms and typical multi-view label propagation algorithms.

\section{Local Linear Single-view Neighbor Reconstruction}

Local linear single-view neighbor reconstruction is based on the local linear neighbor assumption, which insists that each data point can be reconstructed by combining its selected neighbors in the original data space. This assumption is widely used for similarity matrix learning $[24,33,44,8,43]$. The work in $[24,33]$ fixed the number of neighbors and used $l_{2}$-norm in the objective function. Generally, the objective function is shown as Eq.(1).

$$
\begin{aligned}
\varepsilon(W) & =\sum_{i}\left\|x_{i}-\sum_{j: x_{j} \in N\left(x_{i}\right)} W_{j i} x_{j}\right\|^{2} \\
\text { s.t. } & \sum_{j: x_{j} \in N\left(x_{i}\right)} W_{j i}=1, W_{j i} \geq 0
\end{aligned}
$$

In Eq.(1), $W_{j i}=0$ if $x_{j} \notin N\left(x_{i}\right) . N\left(x_{i}\right)$ is the set of $k$ nearest neighbors of $x_{i}$. To avoid using fixed number of neighbors, the work in $[44,8,43]$ minimized the $l_{1}$-norm and was expected to learn a sparse adjacency matrix without fixed neighborhoods for each point. The work in $[8,43]$ tried to optimize Eq.(2), but the weights can not be ensured to be positive. Here $W_{i}$ is the $i$-th column of $W$.

$$
\min _{W}\left\|W_{. i}\right\|_{l_{1}} \quad \text { s.t. } x_{i}=\sum_{j: j \neq i} W_{j i} x_{j}
$$

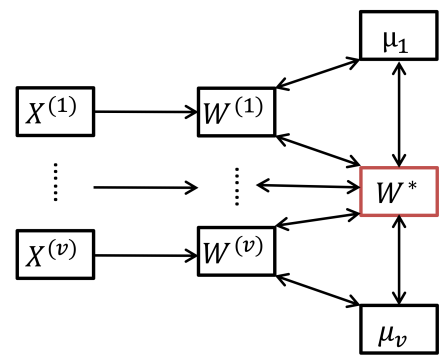

Fig. 1. The interaction between two variables. $X^{(v)}$ is the dataset of $v$-th view. $W^{(v)}$ is the similarity matrix of $v$-th view. $W^{*}$ is the approximated ground truth similarity matrix. $\mu_{v}$ is the weight of $v$-th view.

The algorithm in [44] relaxed the restriction of $[8,43]$ and added the non-negative constraint. Its objective function is Eq.(3).

$$
\min _{W}\left\|x_{i}-\sum_{j: j \neq i} W_{j i} x_{j}\right\|_{2}^{2}+\lambda\left\|W_{. i}\right\|_{l_{1}} \quad \text { s.t. } W_{. i} \geq 0
$$

The similarity matrices learned by Eq.(1) and Eq.(3) are asymmetric. To ensure symmetry, $W_{i j}$ and $W_{j i}$ can be set as $\left(W_{i j}+W_{j i}\right) / 2$.

\section{Local Linear Multi-view Neighbor Reconstruction}

\subsection{Local Linear Multi-view Neighbor Reconstruction}

Data points with multiple views have multiple similarity matrices. As the information in multiple views is compatible and complementary, it is reasonable to learn a unified similarity matrix containing the information of each view.

For the same set of data points, the ground truth similarity between each pair of points should be the same in each view. Intuitively, we seek for an approximated ground truth similarity matrix that consists of the similarity matrix in each view. Given a dataset $X$ with $n_{v}$ views. Let $X^{(v)}=\left[x_{1}^{(v)}, x_{2}^{(v)}, \cdots, x_{n}^{(v)}\right]$ be the set of data points in $v$-th view, where $n$ is the number of data points, $W^{(v)}$ be the similarity matrix in $v$-th view, $W^{*}$ be the approximated ground truth similarity matrix, and $\mu_{v}$ be the weight of $v$-th view. The interactions among $X^{(v)}, W^{(v)}, W^{*}$ and $\mu_{v}$ are shown in Fig.1. $W^{*}$ proportionally learns information from $W^{(v)}$ in each view. $W^{(v)}$ is constructed from $X^{(v)}$ and part of $W^{*}$.

In each view, the appropriate similarity matrix is block-wise with intra-class similarity. Considering the fact that neighborhood contains the local geometrical information [24], we use the local linear neighbor reconstruction method to construc$\mathrm{t}$ similarity matrix in each view. Let $A_{-i}^{(v)}$ be the matrix obtained from $X^{(v)}$ by removing its $i$-th column. We learn the unified similarity between $x_{i}^{(v)}$ and other points by minimizing Eq.(4).

$$
\begin{aligned}
O= & \sum_{v}\left(\left\|x_{i}^{(v)}-A_{-i}^{(v)} W_{. i}^{(v)}\right\|^{2}+2 \lambda\left\|W_{. i}^{(v)}\right\|_{1}\right. \\
+ & \left.\mu_{v}\left\|W_{. i}^{(v)}-W_{. i}^{*}\right\|^{2}\right)+\|\mu\|^{2} \\
\text { s.t. } & W_{. i}^{*} \geq 0, W_{. i}^{(v)} \geq 0 \\
& \|\mu\|_{1}=1, \mu=\left(\mu_{1}, \cdots, \mu_{n_{v}}\right)
\end{aligned}
$$


In Eq.(4), $\|\mu\|^{2}$ is used to avoid overfitting on a single view. The coefficient of $\left\|W_{. i}^{(v)}\right\|_{1}$ is set as $2 \lambda$ to simplify the following optimization.

Eq.(4) extends Eq.(3) to multiple views. The approximated ground truth similarity matrix $W^{*}$ consists of the similarity matrix $W^{(v)}$ in each view. $W^{(v)}$ is sparsely reconstructed with the local linear neighbors of each data point in the original data space. Then, $W^{*}$ implicitly learns the local geometrical information in original data space from multiple views simultaneously.

\subsection{Multiplicative Update Rule}

Eq.(4) is not jointly convex but convex on each variable individually, thus it can be solved by the multiplicative update rule. We present an iterative method, which optimizes one variable while fixing others, for solving it.

\subsubsection{Fixing $W_{. i}^{*}$, $\mu$, compute $W_{. i}^{(v)}$}

For brevity, we only care about terms that are relevant to $W_{. i}^{(v)}$. Then the objective function reduce to Eq.(5).

$$
\begin{aligned}
O_{W_{. i}^{(v)}} & =-2 X_{. i}^{(v) T} A_{-i}^{(v)} W_{. i}^{(v)}+W_{. i}^{(v) T} A_{-i}^{(v) T} A_{-i}^{(v)} W_{. i}^{(v)} \\
& +2 \lambda\left\|W_{. i}^{(v)}\right\|_{1}+\mu_{v}\left\|W_{. i}^{(v)}-W_{. i}^{*}\right\|^{2}
\end{aligned}
$$

Let $\Theta$ be the Lagrange multiplier for $W_{. i}^{(v)}$, then the Lagrangian function of Eq.(5) is Eq.(6).

$$
L=O_{W_{. i}^{(v)}}+\Theta^{T} W_{. i}^{(v)}
$$

The first-order derivative of Eq.(6) is shown in Eq.(7).

$$
L^{\prime}=-2 A_{-i}^{(v) T} X_{. i}^{(v)}+2 A_{-i}^{(v) T} A_{-i}^{(v)} W_{. i}^{(v)}+2 \lambda+2 \mu_{v}\left(W_{. i}^{(v)}-W_{. i}^{*}\right)+\Theta
$$

Then, the update rule of $W_{i}^{(v)}$ can be expressed as follows.

$$
W_{. i}^{(v)} \leftarrow W_{. i}^{(v)} \odot \frac{A_{-i}^{(v) T} X_{. i}^{(v)}+\mu_{\nu} W_{. i}^{*}}{A_{-i}^{(v) T} A_{-i}^{(v)} W_{. i}^{(v)}+\lambda+\mu_{v} W_{. i}^{(v)}}
$$

where $\odot$ is the dot product between two matrices.

\subsubsection{Fixing $W_{. i}^{(v)}, \mu$, compute $W_{i}^{*}$}

Similarly, we get the following objective function Eq.(9). Eq.(9) is a quadratic function of $W_{. i}^{*}$, then we derive Eq.(10).

$$
\begin{gathered}
O_{W_{. i}^{*}}=\sum_{v} \mu_{v}\left\|W_{. i}^{(v)}-W_{. i}^{*}\right\|^{2} \\
W_{. i}^{*}=\frac{\sum_{v} \mu_{v} W_{. i}^{(v)}}{\sum_{v} \mu_{v}}
\end{gathered}
$$

\subsubsection{Fixing $W_{. i}^{*}, W_{. i}^{(v)}$, compute $\mu$}

The objective function with respect to $\mu$ is Eq.(11).

$$
\begin{array}{r}
O_{\mu}=\sum_{v}\left(\mu_{v}\left\|W_{. i}^{(v)}-W_{. i}^{*}\right\|^{2}\right)+\|\mu\|^{2} \\
\text { s.t. }\|\mu\|_{1}=1, \mu=\left(\mu_{1}, \cdots, \mu_{n_{v}}\right)
\end{array}
$$

$\mu$ can be solved by the entropic mirror descent algorithm (EMDA) [3]. EMDA requires the Lipschitz constant as input. For a fixed norm(for example 1-norm), the Lipschitz constant of $\mu$ is as follows.

$$
L_{f}(\mu)=\sup _{\mu}\left\|f^{\prime}(\mu)\right\|_{1}=\sum_{v}\left\|W_{. i}^{(v)}-W_{. i}^{*}\right\|^{2}+2
$$

\subsection{The Algorithm}

We summarize the flow of local linear multi-view neighbor reconstruction (LMNR) algorithm in Algorithm 1. We initially set the element of $W^{(v)}$ and $W^{*}$ as $1 /(n-1)$ and element of $\mu$ as $1 / n_{v}$.

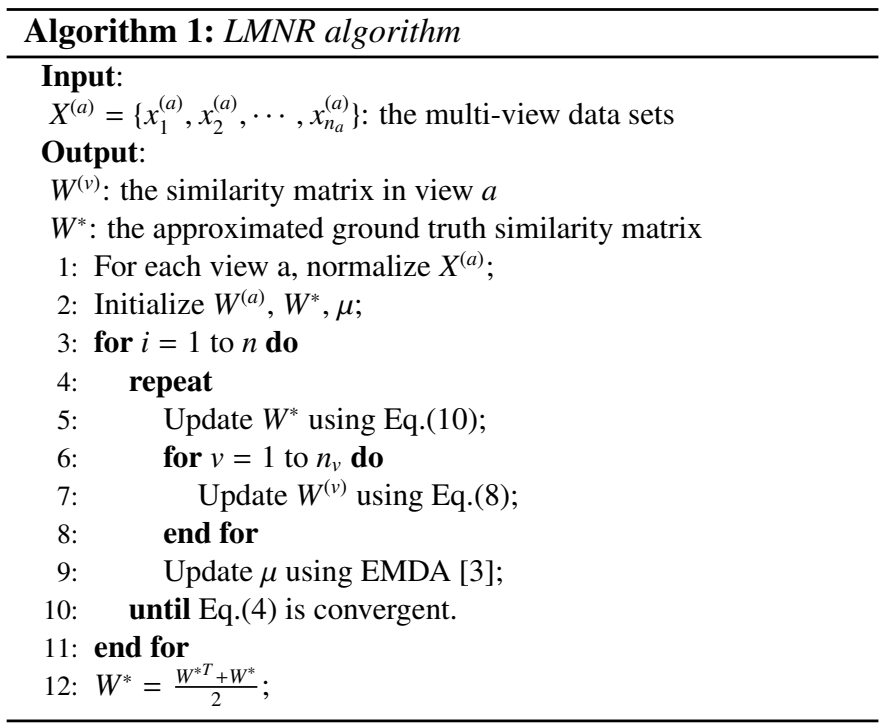

In Fig.2, we demonstrate the benefit of LMNR on a simple synthetic dataset involving 100 samples represented in two views. Fig.2(a) and Fig.2(b) separately show the distribution of the two views. Each view has two clusters and the first 50 samples belong to one cluster (represented by red points) while the others belong to the other cluster (represented by blue points). Specifically, we mark several points to facilitate the discussion. The presentations of the same point in two views are marked by the same shape. In the example, the two clusters seem to be well separated in each view. However, the boundary points (e.g., the point in view 1 marked by square and the point in view 2 marked by circle) are close to some points belonging to both of two clusters. Then, as shown in Fig.2(c) and Fig.2(d) ${ }^{1}$, the intra-cluster similarities may be less than or nearly the same with the inter-cluster similarities. It is hard to separate the boundary points by using the information in a single view. That is, the similarity matrix in each view is not so good to separate the clusters. Fortunately, the two similarity matrices are complementary and capable of identifying individual points, i.e. the points that have high similarities in view 1 have low similarities in view 2 and vice versa (e.g. the point marked by circle and square). By using LMNR, the unified similarities merge together the similarities in two views and subsequently become better to partition clusters. As shown in Fig.2(e), the unified similarity matrix is a proximate block diagonal matrix, so the clusters are well separated.Results on the toy dataset indicate that LMNR leverages the information in multiple views to learn a better unified similarity matrix.

\footnotetext{
${ }^{1}$ The representation of the similarity matrix is bad since the scale of the intra-cluster similarities calculated by Eq.(3) varies greatly. When graphed, the high similarities weaken the representations of low similarities. Actually, most of the intra-cluster similarities are still higher than inter-cluster similarities.
} 


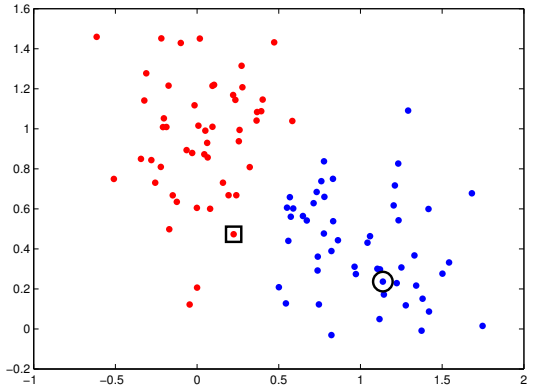

(a) View 1

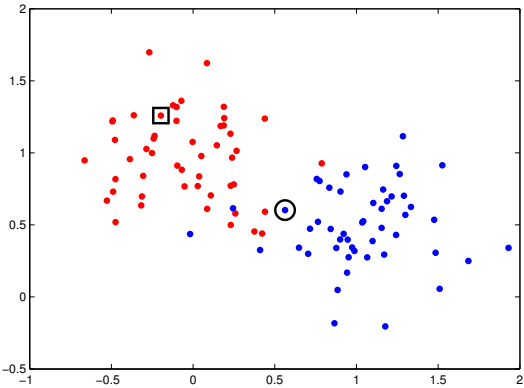

(b) view 2

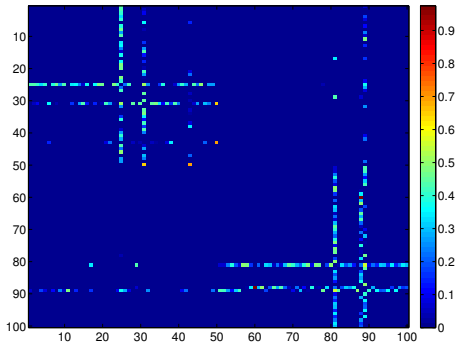

(c) Sparse similarity matrix of view 1

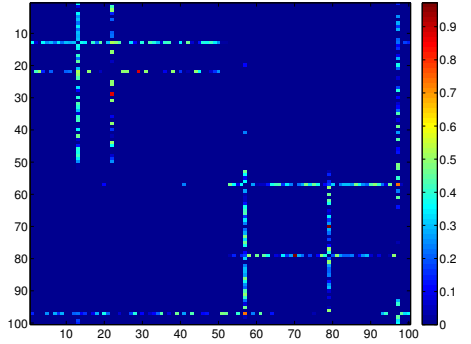

(d) Sparse similarity matrix of view 2

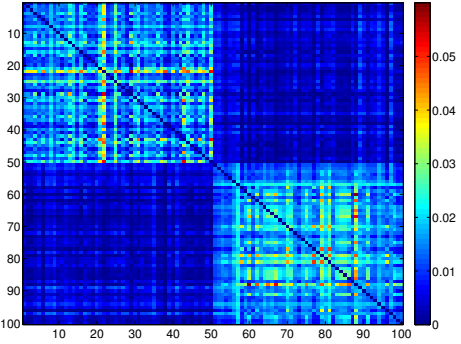

(e) Unified similarity matrix

Fig. 2. Synthetic dataset and the similarity matrix. (a) and (b) are the distribution of the dataset. (c) and (d) are the sparse similarity matrices calculated by Eq.(3). (e) is the unified similarity matrix calculated by LMNR.

\section{Application to Clustering and Label Propagation}

\subsection{Spectral Clustering}

Graph-based clustering, such as spectral clustering [32], aims to partition a dataset into groups according to the pairwise similarities between pairs of data points. We apply the learned similarity matrix on spectral clustering to show its effectiveness. The flow of spectral clustering is shown as follows.

(1) It calculates the Laplacian matrix $L=D^{-1 / 2} W^{*} D^{-1 / 2}$, where $D$ is a diagonal matrix and $D_{i i}=\sum_{j} W_{i j}^{*}$.

(2) It calculates the first $k$ eigenvectors of $L$ and forms $Y$ by the eigenvectors. $k$ is the number of clusters.

(3) It clusters $Y$ with $k$-means into $k$ clusters.

\subsection{Label Propagation}

Label propagation [47] propagates the labels of labeled data to unlabeled data using the similarity matrix. The flow of label propagation is shown as follows.

(1) It calculates the Laplacian matrix $L=D^{-1 / 2} W^{*} D^{-1 / 2}$, where $D$ is a diagonal matrix and $D_{i i}=\sum_{j} W_{i j}^{*}$.

(2) It iterates $F(t+1)=\alpha L F(t)+(1-\alpha) Y$ until convergence, where $\alpha \in(0,1)$ is a parameter previously defined, $Y \in R^{n \times k}$ is the constrained matrix with $Y_{i j}=1$ if $x_{i}$ belongs to $j$-th cluster and $Y_{i j}=0$, otherwise $F(0)=Y$.

(3) Let $F^{*}$ be the limit of $F(t)$. Label $x_{i}$ as a label $y_{i}=$ $\arg \max _{1 \leq j \leq k} F_{i j}^{*}$.

\section{Experiments}

\subsection{Datasets}

We use four datasets to evaluate the effectiveness of LMN$\mathrm{R}$ with application to spectral clustering and label propagation.
UCI Handwritten digits data ${ }^{2}$ consists of 2000 examples, with view-1 being the 76 Fourier coefficients and view- 2 being the 47 Zernike moments. Animal ${ }^{3}$ consists of 30475 images of 50 animals classes with six pre-extracted feature representations for each image. We choose images of 5 animals classes with Local Self-Similarity features and PyramidHOG features. We$\mathrm{bkb}^{4}$ is composed of web pages collected from computer science department of Washington university. A web page is made of two views: content and cites. The documents are described by 1703 words in the content view and 938 links between them in the cites views. M2 4 contains subsets of the NG20 dataset with 3 different preprocessing, supervised mutual information preprocessing, partitioning around medol̈ds preprocessing and unsupervised mutual information preprocessing.

\subsection{Baselines}

LMNR in spectral clustering and label propagation is compared with single-view similarity matrix reconstruction methods: Cos and Sparse, multi-view similarity matrix reconstruction methods: Cos-concat, Sparse-concat, MVSim, Cospectral and MVSpec. Cos calculates cosine similarity on each view; Cos-concat first concatenates all the views, and then calculates cosine similarity on the concatenated data; Sparse calculates the similarity matrix on each view data using method in [44]; Sparse-concat first concatenates all the views, and then calculates the similarity matrix on each view data using method in

\footnotetext{
${ }^{2}$ https://archive.ics.uci.edu/ml/datasets/Multiple+Features

${ }^{3} \mathrm{http}: / /$ attributes.kyb.tuebingen.mpg.de/

${ }^{4}$ http://lig-membres.imag.fr/grimal/data.html
} 
[44]; MVSim [6] simultaneously deals with all the information contained in multi-view datasets by using several instances of an existing co-similarity algorithm; Cospectral [19] constructs the similarity matrix in one view by using the eigenvectors in another view; and MVSpec [30] learns a weighted combination of the given kernels in parallel to clustering.

When applied to spectral clustering, LMNR is also compared with the following multi-view spectral clustering algorithms. Coregspectral [20] co-regularizes the clustering hypotheses; MvSpecCE [37] extends clustering ensembles to multi-view clustering. KCCA [7] is based on kernel canonical correlation analysis. sM-D [14] generates a multipartite graph. CGC [9] minimizes cluster assignment inconsistency with the given cross domain relationships. RMSC [35] learns the consensus transition probability matrix via low-rank and sparse decomposition. To show the influence of $\mu$, we also report the result of LMNR with equally treated views (LMNR- $\mu$ ), i.e. we set the element of $\mu$ as $1 / n_{v}$ for each data point.

When applied to label propagation, LMNR is also compared with one typical multi-view label propagation algorithm: SSMDML [45]. SSM-DML learns multi-view distance metrics from multiple feature sets in the process of label propagation.

Cos, Sparse, MVSim, Cospectral, CGC and sM-D do not have a consensus result, and we report their best single view results. For the baselines, we use the parameters suggested in the original papers. Specifically, each view is equally treated in the baselines except for MVSpec and MvSpecCE. In MVSpec and $\mathrm{MvSpecCE}$, the importance of each view is leveraged by an exponent with power $p=1.5$. In MvSpecCE, we take a coarse grid search for scale parameter in the region $\left[2^{0}, 2^{6}\right]$ with exponent growth 0.5. In MVSIM, the power is set as 0.8 , the percentage of the smallest similarity values is set as 0.4 , the damping parameter is set as 0.5 , the number of iterations is set as 6. In CGC, the regularization parameter is set as 1 . In KCCA, the Gram Schmidt precision parameter is fixed at 0.5. In RMSC, we set the trade-off parameter as 0.005 . In LMNR, $\lambda$ controls the importance of reconstruction and sparsity. To balance the two parts, we set $\lambda$ as 0.5 for all the datasets. For each data point, we set the number of iterations as 10 since Eq.(4) generally converges in limited steps $(; 10)$. In label propagation, we set $\alpha$ as 0.2 . To avoid randomness, we conduct the algorithms 20 runs with different initializations and report the average results.

\subsection{Evaluation Metrics}

We use accuracy (ACC) and normalized mutual information (NMI) [39] to measure the performance.

\subsubsection{Accuracy}

The accuracy (ACC) measure calculates the largest rate of correct assignation by corresponding each data to the right cluster. Denote $c_{i}$ as the label of an algorithm and $l_{i}$ as the true label of $x_{i}$. ACC is defined as:

$$
A C C=\frac{\sum_{i=1}^{n} \delta\left(l_{i}, \operatorname{map}\left(c_{i}\right)\right)}{n}
$$

where $\delta(x, y)$ is the indicator function, and $n$ is the total number of objects, $\operatorname{map}\left(c_{i}\right)$ is the mapping function that permutes the clustering labels to match the ground truth data labels.

\subsubsection{Normalized Mutual Information}

The normalized mutual information(NMI) indicates the shared statistics between the label of an algorithm and the true label. Given the clustering result, NMI is computed as follows.

$$
N M I=\frac{\sum_{c_{i} \in C, c_{j}^{\prime} \in C^{\prime}} p\left(c_{i}, c_{j}^{\prime}\right) \cdot \log \frac{p\left(c_{i}, c_{j}^{\prime}\right)}{p\left(c_{i}\right) \cdot p\left(c_{j}^{\prime}\right)}}{\sqrt{\sum_{c_{i} \in C} p\left(c_{i}\right) \log p\left(c_{i}\right) \sum_{c_{j}^{\prime} \in C^{\prime}} p\left(c_{j}^{\prime}\right) \log p\left(c_{j}^{\prime}\right)}}
$$

where $p\left(c_{i}\right)$ denotes the probabilities that an object belongs to the clusters $c_{i}$, and $p\left(c_{i}, c_{j}^{\prime}\right)$ is the joint probability that the object belongs to the clusters $c_{i}$ as well as $c_{j}^{\prime}$ at the same time.

\subsection{Spectral Clustering Results}

Table 1 shows the results of spectral clustering. The sixth column and the last column separately show the average AC$\mathrm{C}$ and NMI of every algorithm over the four datasets. Generally, Cos-concat and Sparse-concat have no advantage over their single view counterparts, which shows that simply concatenating all the features together may lose information of single view. The multi-view baselines perform better than Cos and Sparse in most cases, which indicates that combining information in multiple views improves the clustering performance. LMNR always performs better than the other single-view and multi-view similarity matrix reconstruction methods, and it outperforms the multi-view spectral clustering algorithms in most cases. As exceptional cases, for Handwritten, LMNR performs not as well as Cospectral in terms of ACC; for Webkb, LMNR performs worse than CGC and sM-D in terms of ACC. However, LMNR performs the best on all the datasets in terms of $\mathrm{N}$ MI. In addition, LMNR performs slightly better than LMNR- $\mu$, which shows that the strategy of adjusting $\mu$ is effective. Overall, the average performance of LMNR is the best in terms of both ACC and NMI. We can conclude that LMNR performs the best among all the tested methods, which is due to integrating original local linear neighbor information from multiple views.

\subsection{Label Propagation Results}

Fig. 3 shows the results of label propagation in terms of ACC and NMI. For each dataset, we randomly label $6 \% \sim 20 \%$ data points. From the results it can be seen that label propagation based on LMNR performs better than label propagation based on the other similarity matrix reconstruction methods, and the multi-view label propagation method, SSM-DML. The performance of LMNR based label propagation increases stably when increasing the percent of labeled data. Increasing the number of labeled data also generally improves the performance of other algorithms, but they increase slower than LMNR. This is because that label propagation mainly depends on the local geometrical structure, which could be represented by local linear neighbors. LMNR not only contains local linear neighbor information but also integrates information from multiple views, while other methods contain only one kind of information. 
Table 1. Accuracy (ACC) and normalized mutual information (NMI) on all datasets. The best result of each column is highlighted in boldface.

\begin{tabular}{|c|c|c|c|c|c|c|c|c|c|c|}
\hline \multirow{2}{*}{ Algorithm } & \multicolumn{5}{|c|}{$\mathrm{ACC}(\%)$} & \multicolumn{5}{|c|}{ NMI $(\%)$} \\
\hline & Handwritten & Animal & Webkb & M2 & Avg. & Handwritten & Animal & Webkb & M2 & Avg. \\
\hline Cos & $53.96 \pm 0.20$ & $35.59 \pm 0.00$ & $51.57 \pm 0.50$ & $93.60 \pm 0.00$ & 58.68 & $49.75 \pm 0.16$ & $10.44 \pm 0.00$ & $31.33 \pm 0.36$ & $65.95 \pm 0.00$ & 39.37 \\
\hline Cos-concat & $65.89 \pm 0.08$ & $39.35 \pm 0.05$ & $51.54 \pm 0.50$ & $54.00 \pm 0.00$ & 52.69 & $58.16 \pm 0.18$ & $13.93 \pm 0.02$ & $32.57 \pm 0.50$ & $1.59 \pm 0.00$ & 26.56 \\
\hline Sparse & $26.16 \pm 2.28$ & $33.88 \pm 0.27$ & $54.35 \pm 1.93$ & $95.00 \pm 0.00$ & 52.35 & $23.33 \pm 1.61$ & $10.63 \pm 0.02$ & $34.10 \pm 1.68$ & $72.03 \pm 0.00$ & 35.02 \\
\hline Sparse-concat & $13.89 \pm 0.14$ & $39.38 \pm 0.00$ & $54.24 \pm 1.81$ & $98.20 \pm 0.00$ & 51.43 & $7.85 \pm 0.27$ & $13.76 \pm 0.00$ & $33.81 \pm 0.70$ & $87.14 \pm 0.00$ & 35.64 \\
\hline MVSim & $28.35 \pm 1.02$ & $41.59 \pm 0.00$ & $47.85 \pm 0.10$ & $96.40 \pm 0.00$ & 53.55 & $27.73 \pm 0.60$ & $13.91 \pm 0.20$ & $10.87 \pm 0.01$ & $78.14 \pm 0.00$ & 32.66 \\
\hline Coregspectral & $59.02 \pm 3.35$ & $38.46 \pm 0.63$ & $44.39 \pm 3.20$ & $96.00 \pm 0.13$ & 59.47 & $53.96 \pm 1.46$ & $13.36 \pm 0.10$ & $23.34 \pm 2.50$ & $75.89 \pm 0.58$ & 41.64 \\
\hline Cospectral & $68.99 \pm 0.14$ & $41.25 \pm 0.02$ & $51.16 \pm 6.97$ & $95.20 \pm 0.00$ & 64.15 & $59.95 \pm 0.06$ & $12.36 \pm 0.00$ & $29.82 \pm 6.15$ & $79.57 \pm 0.00$ & 45.42 \\
\hline MVSpec & $54.35 \pm 0.00$ & $36.43 \pm 0.00$ & $56.96 \pm 0.00$ & $52.00 \pm 0.00$ & 49.93 & $50.45 \pm 0.00$ & $11.13 \pm 0.00$ & $35.49 \pm 0.00$ & $0.45 \pm 0.00$ & 24.38 \\
\hline CGC & $36.55 \pm 2.52$ & $33.74 \pm 2.38$ & $62.09 \pm 5.36$ & $75.81 \pm 5.17$ & 52.05 & $33.57 \pm 2.25$ & $9.73 \pm 0.95$ & $35.63 \pm 2.51$ & $30.48 \pm 9.98$ & 27.35 \\
\hline MvSpecCE & $54.65 \pm 0.00$ & $36.43 \pm 0.00$ & $42.61 \pm 0.00$ & $73.60 \pm 0.00$ & 51.82 & $50.10 \pm 0.00$ & $10.83 \pm 0.00$ & $7.70 \pm 0.00$ & $17.77 \pm 0.00$ & 21.60 \\
\hline KCCA & $26.88 \pm 1.08$ & $36.63 \pm 0.00$ & $42.61 \pm 0.00$ & $62.80 \pm 0.00$ & 42.23 & $25.46 \pm 0.14$ & $11.06 \pm 0.00$ & $9.78 \pm 0.00$ & $14.03 \pm 0.00$ & 15.08 \\
\hline sM-D & $66.15 \pm 3.87$ & $35.95 \pm 0.19$ & $62.52 \pm 0.79$ & $95.50 \pm 0.00$ & 65.03 & $58.02 \pm 1.36$ & $12.31 \pm 0.18$ & $38.59 \pm 1.77$ & $73.71 \pm 0.00$ & 45.66 \\
\hline RMSC & $62.28 \pm 3.69$ & $40.88 \pm 2.08$ & $40.93 \pm 4.05$ & $96.80 \pm 0.00$ & 60.22 & $55.74 \pm 2.12$ & $14.19 \pm 1.42$ & $18.39 \pm 3.66$ & $80.97 \pm 0.00$ & 42.32 \\
\hline LMNR- $\mu$ & $64.87 \pm 2.41$ & $40.41 \pm 0.00$ & $56.11 \pm 5.10$ & $99.40 \pm 0.00$ & 65.20 & $62.37 \pm 0.84$ & $14.23 \pm 0.00$ & $43.37 \pm 0.13$ & $94.76 \pm 0.00$ & 53.68 \\
\hline LMNR & $65.01 \pm 1.17$ & $42.41 \pm 0.00$ & $56.61 \pm 5.03$ & $99.40 \pm 0.00$ & 65.86 & $62.46 \pm 0.19$ & $14.23 \pm 0.00$ & $43.38 \pm 0.13$ & $94.76 \pm 0.00$ & 53.71 \\
\hline
\end{tabular}

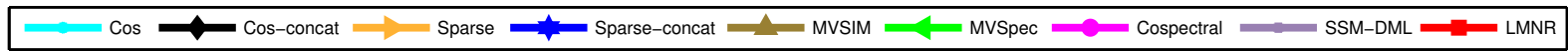

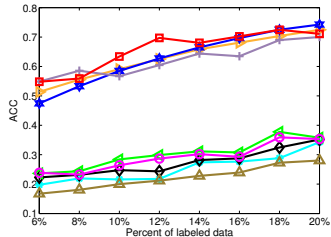

(a) Handwritten-ACC

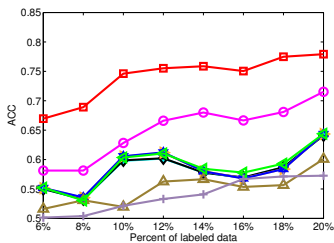

(e) Webkb-ACC

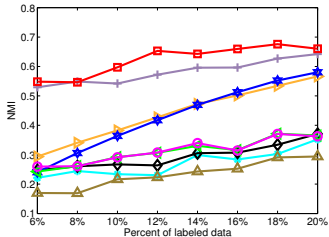

(b) Handwritten-NMI

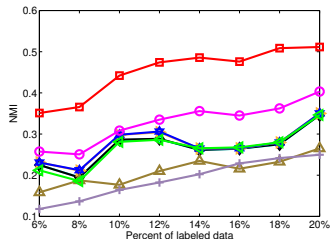

(f) Webkb-NMI

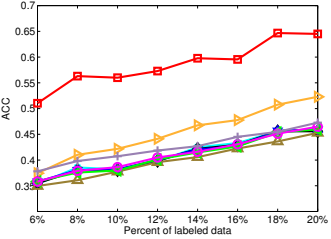

(c) Animal-ACC

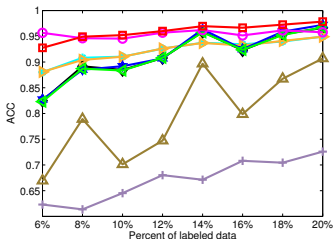

(g) M2-ACC

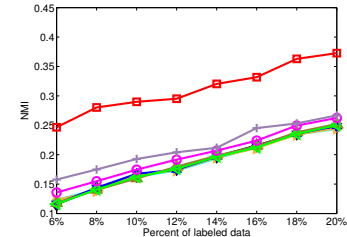

(d) Animal-NMI

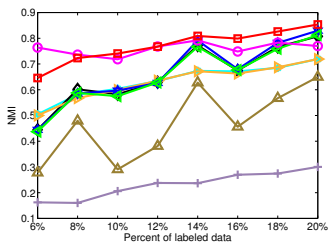

(h) M2-NMI

Fig. 3. Accuracy (ACC) and normalized mutual information (NMI) of label propagation on all datasets. The horizontal axis is the percent of labeled data points and the vertical axis shows the corresponding performances.

\section{Related Work}

\subsection{Multi-view Metric Learning}

Multi-view metric learning constructs embedding projection$\mathrm{s}$ from multi-view data into a shared feature space.

Following the classical principle of pulling samples together if they are related and pushing them apart if they are not, Quadrianto and Lampert [23] constructed Euclidean distance that provided a meaningful within-view as well as betweenview similarity. Based on the theory of graph-based semisupervised learning, Yu et al. [45] learned the multi-view distance metrics from multiple feature sets and the labeled characters. Zhai et al. [46] learned the multi-view metric with part of labeled sets and considered global consistency and local smoothness. Cui et al. [11] proposed a pairwise-constrained multiple metric learning method to effectively integrate the face region descriptors of all blocks. Hu et al. [16] maximized the correlations of different feature representations of each sample.
Wu and Shah [34] proposed a class-dependent distance between two multi-view data points by weighting the data in each view upon the classification impact of the specific viewpoint to the corresponding action class. Xie and Xing [36] embedded data of arbitrary modalities into a single latent space, and learned the distance metric by minimizing the distance between similar pairs whereas maximizing the distance between dissimilar.

Spectral clustering is a graph based unsupervised method, so we do not compare it with these Euclidean distance based methods and semi-supervised methods. For label propagation, we choose to compare with a typical multi-view label propagation method [45].

\subsection{Multiple Kernel Learning}

Multiple kernel learning (MKL) was first proposed in [21], where it considers conic combinations of kernel matrices. Later methods find either a linear combination or a nonlinear combination of base kernels. 
Sonnenburg et al. [28] cast the method in [21] into a SemiDefinite Programming problem and used $l_{1}$ norm to regularize multiple kernels. Xu et al. [41] introduced smoothness to the optimization procedure. Kowalski et al. [18] involved a misclassification loss term and a regularization term that was expressed by means of a mixed norm. Xu et al. [40] formulated a closed-form solution for optimizing the kernel weights on basis of the equivalence between group-lasso and MKL. Yan et al. [42] generalized multiple kernel Fisher discriminant analysis such that the kernel weights can be regularised with $l_{p}$-norm for any $p \geq 1$. Tzortzis and Likas [30] learned a weighted combination of the given kernels in parallel to clustering. Guo et al. [15] learned a linear combination of multiple kernels and determined the coefficient by minimizing the kernel alignment between the learned kernel and the kernel of each view.

The methods in $[31,10]$ tried to find a polynomial combination of the base kernels. Jagarlapudi et al. [17] employed $l_{\infty}$ regularization for promoting combinations at the component level and $l_{1}$ regularization for promoting sparsity among kernels in each component. Aflalo et al. [1] employed $l_{1}$-norm regularization for promoting sparsity within RKHS norms of each group and $l_{p}$-norm, $p \geq 2$, regularization for promoting non-sparse combinations across groups. Bach [2] used $l_{1}$-norm inside the feature space.

\section{Conclusion}

Similarity matrix is one of the frequently used technique in data analysis. For data with multiple views, it is better to learn a similarity matrix containing information in all views. In this paper, we propose to construct a similarity matrix by using local linear neighbors in multiple views. In each view, the similarity matrix can be reconstructed with the weight of neighbors. In multiple views, we seek for an approximated ground truth similarity matrix which is proportionally consistent with the similarity matrix in each view. The learned similarity matrix can be used for spectral clustering, label propagation and other graph based learning algorithms. Results on several datasets have shown that the learned similarity matrix enhances the performance of both spectral clustering and label propagation.

\section{Acknowledgments}

This work was supported by National Science Foundation of China (No. 61272374,61300190,61428202) and National High Technology Research and Development Program (863 Program) of China (No. 2015AA015403).

\section{References}

[1] Aflalo, J., Ben-Tal, A., Bhattacharyya, C., Nath, J.S., Raman, S., 2011. Variable sparsity kernel learning. J. Mach. Learn. Res. 12, 565-592.

[2] Bach, F.R., 2009. Exploring large feature spaces with hierarchical multiple kernel learning, in: Advances in neural information processing systems, pp. 105-112.

[3] Beck, A., Teboulle, M., 2003. Mirror descent and nonlinear projected subgradient methods for convex optimization. Oper. Res. Lett. 31, 167175 .
[4] Becker, S., Hinton, G.E., 1989. Spatial coherence as an internal teacher for a neural network. Technical Report CRG-TR-89-7, Department of Computer Science, University of Toronto, Ontario .

[5] Becker, S., Hinton, G.E., 1992. Self-organizing neural network that discovers surfaces in random-dot stereograms. Nature. 355, 161-163.

[6] Bisson, G., Grimal, C., 2012. Co-clustering of multi-view datasets: A parallelizable approach., in: The 10th IEEE International Conference on Data Mining, pp. 828-833.

[7] Blaschko, M.B., Lampert, C.H., 2008. Correlational spectral clustering, in: IEEE Conference on Computer Vision and Pattern Recognition, IEEE. pp. $1-8$.

[8] Cheng, H., Liu, Z., Yang, J., 2009. Sparsity induced similarity measure for label propagation, in: 2009 IEEE 12th International Conference on Computer Vision, IEEE. pp. 317-324.

[9] Cheng, W., Zhang, X., Guo, Z., Wu, Y., Sullivan, P.F., Wang, W., 2013. Flexible and robust co-regularized multi-domain graph clustering, in: Proceedings of the 19th ACM SIGKDD international conference on Knowledge discovery and data mining, ACM. pp. 320-328.

[10] Cortes, C., Mohri, M., Rostamizadeh, A., 2009. Learning non-linear combinations of kernels, in: Advances in neural information processing systems, pp. 396-404.

[11] Cui, Z., Li, W., Xu, D., Shan, S., Chen, X., 2013. Fusing robust face region descriptors via multiple metric learning for face recognition in the wild, in: 2013 IEEE Conference on Computer Vision and Pattern Recognition, IEEE. pp. 3554-3561.

[12] De Sa, V.R., 1994. Minimizing disagreement for self-supervised classification, in: Proceedings of the 1993 Connectionist Models Summer School, Psychology Press. p. 300.

[13] De Sa, V.R., Ballard, D.H., 1998. Category learning through multimodality sensing. Neural Comput. 10, 1097-1117.

[14] De Sa, V.R., Gallagher, P.W., Lewis, J.M., Malave, V.L., 2010. Multiview kernel construction. Mach. Learn. 79, 47-71.

[15] Guo, D., Zhang, J., Liu, X., Cui, Y., Zhao, C., 2014. Multiple kernel learning based multi-view spectral clustering, in: 22nd International Conference on Pattern Recognition, IEEE. pp. 3774-3779.

[16] Hu, J., Lu, J., Yuan, J., Tan, Y.P., 2015. Large margin multi-metric learning for face and kinship verification in the wild, in: 12th Asian Conference on Computer Vision. Springer, pp. 252-267.

[17] Jagarlapudi, S.N., Dinesh, G., Raman, S., Bhattacharyya, C., Ben-Tal, A., Kr, R., 2009. On the algorithmics and applications of a mixed-norm based kernel learning formulation, in: Advances in neural information processing systems, pp. 844-852.

[18] Kowalski, M., Szafranski, M., Ralaivola, L., 2009. Multiple indefinite kernel learning with mixed norm regularization, in: Proceedings of the 26th Annual International Conference on Machine Learning, ACM. pp. $545-552$.

[19] Kumar, A., Daumé, H., 2011. A co-training approach for multi-view spectral clustering, in: Proceedings of the 28th International Conference on Machine Learning, pp. 393-400.

[20] Kumar, A., Rai, P., Daume, H., 2011. Co-regularized multi-view spectral clustering, in: Advances in Neural Information Processing Systems, pp. 1413-1421.

[21] Lanckriet, G.R., Cristianini, N., Bartlett, P., Ghaoui, L.E., Jordan, M.I., 2004. Learning the kernel matrix with semidefinite programming. J. Mach. Learn. Res. 5, 27-72.

[22] Li, Y., Nie, F., Huang, H., Huang, J., 2015. Large-scale multi-view spectral clustering via bipartite graph, in: Proceedings of the Twenty-Ninth AAAI Conference on Artificial Intelligence, pp. 2750-2756.

[23] Quadrianto, N., Lampert, C.H., 2011. Learning multi-view neighborhood preserving projections, in: Proceedings of the 28th International Conference on Machine Learning, pp. 425-432.

[24] Roweis, S.T., Saul, L.K., 2000. Nonlinear dimensionality reduction by locally linear embedding. Science. 290, 2323-2326.

[25] de Sa, V.R., 1994. Learning classification with unlabeled data, in: Advances in neural information processing systems, pp. 112-119.

[26] de Sa, V.R., Ballard, D.H., 1993. Self-teaching through correlated input, in: Computation and neural systems. Springer, pp. 437-441.

[27] Schmidhuber, J., Prelinger, D., 1993. Discovering predictable classifications. Neural Comput. 5, 625-635.

[28] Sonnenburg, S., Rätsch, G., Schäfer, C., 2006. A general and efficient multiple kernel learning algorithm, in: Advances in neural information processing systems, pp. $1273 \mathrm{C}-1280$. 
[29] Sun, S., 2013. A survey of multi-view machine learning. Neural Comput. Appl. 23, 2031-2038.

[30] Tzortzis, G., Likas, A., 2012. Kernel-based weighted multi-view clustering., in: 12th IEEE International Conference on Data Mining, pp. 675684.

[31] Varma, M., Babu, B.R., 2009. More generality in efficient multiple kernel learning, in: Proceedings of the 26th Annual International Conference on Machine Learning, ACM. pp. 1065-1072.

[32] Von Luxburg, U., 2007. A tutorial on spectral clustering. Stat. Comput. 17, 395-416.

[33] Wang, F., Zhang, C., 2008. Label propagation through linear neighborhoods. IEEE Trans. Knowl. Data Eng. 20, 55-67.

[34] Wu, X., Shah, S.K., 2014. Regularized multi-view multi-metric learning for action recognition, in: International Conference on Pattern Recognition, pp. 471-476.

[35] Xia, R., Pan, Y., Du, L., Yin, J., 2014. Robust multi-view spectral clustering via low-rank and sparse decomposition, in: AAAI Conference on Artificial Intelligence, pp. 2149-2155.

[36] Xie, P., Xing, E.P., 2013. Multi-modal distance metric learning, in: Proceedings of the Twenty-Third international joint conference on Artificial Intelligence, AAAI Press. pp. 1806-1812.

[37] Xie, X., Sun, S., 2013. Multi-view clustering ensembles, in: International Conference on Machine Learning and Cybernetics, IEEE. pp. 51-56.

[38] Xu, C., Tao, D., Xu, C., 2013. A survey on multi-view learning. CoRR abs/1304.5634.

[39] Xu, W., Liu, X., Gong, Y., 2003. Document clustering based on nonnegative matrix factorization, in: Proceedings of the 26th annual international ACM SIGIR conference on Research and development in informaion retrieval, pp. 267-273.

[40] Xu, Z., Jin, R., Yang, H., King, I., Lyu, M.R., 2010a. Simple and efficient multiple kernel learning by group lasso, in: Proceedings of the 27th international conference on machine learning, pp. 1175-1182.

[41] Xu, Z., Jin, R., Zhu, S., Lyu, M.R., King, I., 2010b. Smooth optimization for effective multiple kernel learning, in: Proceedings of the TwentyFourth AAAI Conference on Artificial Intelligence, pp. 637-642.

[42] Yan, F., Mikolajczyk, K., Barnard, M., Cai, H., Kittler, J., 2010. Lp norm multiple kernel fisher discriminant analysis for object and image categorisation, in: 2010 IEEE Conference on Computer Vision and Pattern Recognition, IEEE. pp. 3626-3632.

[43] Yan, S., Wang, H., 2009. Semi-supervised learning by sparse representation., in: Proceedings of the SIAM International Conference on Data Mining, SIAM. pp. 792-801.

[44] Yang, N., Sang, Y., He, R., Wang, X., 2010. Label propagation algorithm based on non-negative sparse representation, in: Life System Modeling and Intelligent Computing. Springer, pp. 348-357.

[45] Yu, J., Wang, M., Tao, D., 2012. Semisupervised multiview distance metric learning for cartoon synthesis. IEEE Trans. Image Process. 21, 4636-4648.

[46] Zhai, D., Chang, H., Shan, S., Chen, X., Gao, W., 2012. Multiview metric learning with global consistency and local smoothness. ACM Trans. Intell. Syst. Technol. 3, 53.

[47] Zhou, D., Bousquet, O., Lal, T.N., Weston, J., Schölkopf, B., 2004. Learning with local and global consistency, in: Advances in neural information processing systems, pp. 321-328. 